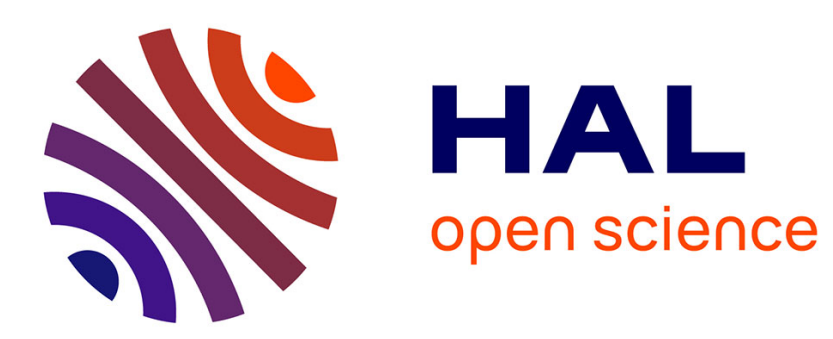

\title{
Les vertus stratégiques de la discrétion des services documentaires Théories et illustrations
}

\author{
Isabelle Le Bis, Béatrice Vacher
}

\section{To cite this version:}

Isabelle Le Bis, Béatrice Vacher. Les vertus stratégiques de la discrétion des services documentaires Théories et illustrations. Documentaliste - Sciences de l'Information, 2006, 10.3917/docsi.433.0200 . halshs-02916221

\section{HAL Id: halshs-02916221 https://shs.hal.science/halshs-02916221}

Submitted on 17 Aug 2020

HAL is a multi-disciplinary open access archive for the deposit and dissemination of scientific research documents, whether they are published or not. The documents may come from teaching and research institutions in France or abroad, or from public or private research centers.
L'archive ouverte pluridisciplinaire HAL, est destinée au dépôt et à la diffusion de documents scientifiques de niveau recherche, publiés ou non, émanant des établissements d'enseignement et de recherche français ou étrangers, des laboratoires publics ou privés. 


\title{
Les vertus stratégiques de la discrétion des services documentaires Théories et illustrations
}

\author{
Par Isabelle Le Bis et Béatrice Vacher \\ Revue Documentaliste - Sciences de l'information 2006, vol. 43, n³-4, p. 200-208
}

\section{Résumé court}

Cet article présente une étude de l'activité documentaire au sein d'un laboratoire scientifique et les actions de restructuration qui y ont été menées. Cette étude de cas est prétexte à s'interroger sur les raisons de l'absence de visibilité des services documentaires et sur le mauvais usage et la faible considération qui en résultent. Pour les auteures, les documentalistes effectuent trop discrètement les tâches matérielles qui leur incombent en mettant en avant la plus valorisante fonction médiation. Or c'est en tirant parti de l'invisible matérialité de leur travail quotidien qu'ils peuvent recentrer la documentation au cœur du métier de l'organisation et s'imposer au sein de sa stratégie.

\section{Résumé long}

Cet article présente une étude de l'activité documentaire au sein d'un laboratoire scientifique et les actions de restructuration qui y ont été menées. Cette étude de cas est prétexte, pour Isabelle Le Bis et Béatrice Vacher, à s'interroger sur les raisons de l'absence de visibilité des services documentaires et sur le mauvais usage et la faible considération qui en résultent.

Pourquoi, alors que les compétences et les connaissances nécessaires à l'exercice de la fonction (organisation, esprit de synthèse, ouverture d'esprit, flexibilité) sont celles que l'on réclame en entreprise, les professionnels de l'I-D ont-ils tant de mal à intégrer le cœur d'activité des entités qui les emploient ? Pour les auteures, c'est parce que les documentalistes effectuent trop discrètement les tâches matérielles qui leur incombent (collecte, rangement, saisie) en s'attachant à ne montrer de leur travail que la fonction médiation qu'ils estiment plus valorisante. Or c'est précisément en tirant parti de l'invisible matérialité de leur travail quotidien qu'ils peuvent recentrer la documentation dans le cœur du métier de l'organisation où ils exercent et s'immiscer au sein de sa stratégie.

Dans le cas du laboratoire présenté ici en exemple, ce recentrage a été possible grâce à une forte implication de la direction, à la mise en place d'une véritable politique documentaire, à la collaboration régulière entre les professionnels de l'information et les chercheurs et au partage des tâches courantes.

\section{Introduction}

PAR QUel etRANGe PARADOXE, dans la société de l'information, méconnaît-on à ce point les professionnels de l'information que sont les documentalistes? Les services documentaires n'intéressent les manageurs que lorsqu'ils coûtent trop chers ou qu'ils dysfonctionnent. Le même constat peut être fait pour les métiers de service dans une société où ce qui compte est autant, sinon plus, le service que le produit. Ces métiers seraientils inutiles, quoi qu'on en dise ? Nous proposons de poser la question autrement, à partir du constat récurrent de l'invisibilité de ce travail pourtant très matériel et en relation directe avec un public.

Tel est en effet le problème : dans une société qui promeut la réflexion, les diplômes et la rationalité, il n'est guère valorisant de se consacrer aux tâches de rangement, classement et saisie, qui plus est au service d'utilisateurs toujours pressés et pas toujours reconnaissants. Inhérente au service documentaire, la 
" manutention de l'information ${ }^{1}$ " est cachée au profit de la noble médiation, même si celle-ci a du mal à se concrétiser. Penser la reconnaissance du métier au niveau stratégique de l'organisation dans laquelle il s'insère, c'est tirer le meilleur parti des services proposés, c'est recentrer la documentation dans le cœur du métier de l'organisation sans pour autant lui faire perdre la discrétion qui la caractérise. Est-ce possible? Comment?

C'est ce que nous proposons d'illustrer à travers un exemple significatif : un important laboratoire de recherche en sciences humaines et sociales (nommé ci-dessous "le Labo ") est à la fois en phase de repositionnement stratégique et en situation de réduction budgétaire. La bibliothèque et les services associés sont-ils vraiment indispensables? En enquêtant au plus près du travail du chercheur, principal acteur du Labo, nous avons aidé ce dernier à répondre (par l'affirmative) et à mobiliser ces services documentaires pour cadrer sa stratégie à long terme.

Nous présentons notre approche en trois points: dans quelle mesure la documentation est une activité invisible (1), ce que l'on retrouve dans de nombreuses activités de service (2), et comment dépasser ces contraintes : une étude de cas et ses éléments théoriques sous-jacents (3).

\section{Une activité invisible et pourtant essentielle}

Parce que le mot documentation est ambigu (il ne désigne pas la même chose selon les métiers, les statuts sociaux, le niveau culturel), l'activité documentaire, lorsqu'elle se situe hors du cadre rassurant d'une bibliothèque, d'un dépôt d'archives ou d'un service ministériel, demeure difficile à définir. Ses responsables hiérarchiques la cernent mal, ont tendance à la traiter en gestionnaires, à distance, en déléguant la fonction. On ne s'en préoccupe souvent que pour faire des gains de productivité ${ }^{2}$.

La fonction documentaire relève de trois familles de métiers - documentalistes, bibliothécaires, archivistes qui illustrent chacune une approche du traitement des documents, entendus au sens large. Dans tout ce qui suit, nous entendons par "bibliothèque " une bibliothèque de recherche ou d'entreprise, à usage spécialisé ; nous excluons volontairement la question des bibliothèques de lecture publique, relativement éloignée de notre problématique.

Bibliothécaires et archivistes ont de longue date acquis une légitimité professionnelle et scientifique. En témoignent, par exemple, le "corps scientifique des bibliothèques » et celui des conservateurs du patrimoine (dont les conservateurs d'archives), corps formés dans des grands établissements ${ }^{3}$. Dans l'esprit du public, les conservateurs des bibliothèques sont des érudits, tradition remontant pour les bibliothécaires aux Bénédictins de la Congrégation de Saint-Maur, à la recherche historique et à la collecte des sources de l'histoire de France [2]. La tradition érudite et humaniste des archivistes n'a rien à leur envier puisqu'on l'évoque déjà du temps du premier humanisme français sous le règne de Charles VI (1380-1422), où des intellectuels érudits ont tenu la charge de notaires et secrétaires du roi ou de gardes du trésor des chartes.

$1 \quad$ Ce terme, qui met en valeur la matérialité intrinsèque des activités d'information, a été énoncé pour la première fois en 1996 et repris en 1998 [29].

2 Ce fut en particulier la raison de notre première intervention commune pour un groupe industriel engagé dans deux démarches conjointes : la redéfinition de ses fonctions centrales et la recherche de performance pour ses fonctions de service. Le rapport de l'étude est actuellement sous contrainte de confidentialité.

3 Décret $n^{\circ} 92-26$ du 9 janvier 1992 modifié portant statut particulier du corps des conservateurs des bibliothèques et du corps des conservateurs généraux des bibliothèques (version consolidée au 18 octobre 2001) et décret n 90-404 du 16 mai 1990 modifié portant statut particulier du corps des conservateurs du patrimoine (version consolidée au 22 décembre 2001). Depuis 1992, les conservateurs de bibliothèques sont tous formés à l'ENSSIB, ceux du patrimoine à l'Institut national du patrimoine. 
Le bibliothécaire reste malgré tout la figure la plus connue : il travaille dans un établissement bien identifié, un lieu où l'on peut toucher, voir ou entendre des collections de documents (entités matérielles comme les ouvrages, disques ou cédéroms) décrits dans un catalogue. Il a une vue d'ensemble du fonds documentaire qu'il gère et met à disposition. Pendant de longues années, c'est avant tout quelqu'un chargé de conserver les collections, un conservateur au sens strict. Conservateur encore - avant de se muer en records manager l'archiviste qui a la charge des dossiers de travail constitués au cours d'une activité professionnelle, qui trie, élimine et décide de la conservation à but historique d'échantillons, de séries de documents ou de documents mémorables.

Plus ambiguë est la situation du documentaliste. La mise en place de formations diplômantes, à l'Institut national des techniques de la documentation (INTD) au sein du Conservatoire national des arts et métiers, dès 1950, puis à I'Université dans le milieu des années soixante, a été difficile et semée d'embûches. Même s'il s'agissait de former en priorité des interlocuteurs documentaires privilégiés des entreprises, nombre de documentalistes ont exercé et exercent encore leur activité dans les diverses fonctions publiques. Contrairement à leurs collègues bibliothécaires ou archivistes qui évoluent entre pairs, les documentalistes exercent souvent seuls ou en petites unités au sein d'une institution qui en attend du profit. C'est dire qu'ils ne peuvent espérer de reconnaissance que du milieu qui les emploie, tantôt intéressé, tantôt un peu hostile.

Le travail du documentaliste consiste à réunir des documents et des informations pour répondre au besoin précis d'un ou de plusieurs utilisateurs. II ressemble ainsi à celui du bibliothécaire puisqu'il consiste à entretenir un fonds documentaire et des outils de recherche adaptés. Mais la fonction de médiation prend le pas sur le traitement de l'information : formation, démonstration, transfert de technicité, etc. Hélas pour son image, le métier de documentaliste ne peut revendiquer une aussi « noble et ancienne filiation » que les conservateurs $\mathrm{d}^{\prime}$ 'archives et des bibliothèques. D'invention trop récente (le $X X^{\mathrm{e}}$ siècle) pour une culture française qui n'accorde de valeur qu'à l'ancien, il est irrémédiablement entaché dans l'esprit des manageurs d'un rapport pas si lointain avec les fonctions de secrétariat. Cette parenté imposée de l'extérieur a été rejetée avec force par les documentalistes qui ont sans cesse voilé leur activité matérielle tout en travaillant à créer une " troisième voie » entre archives et bibliothèques.

C'est ce dont témoigne I'histoire de I'INTD, placé de 1958 à 1980 sous la direction d'un inspecteur général des bibliothèques, puis, de 1980 à 1997, sous celle d'un archiviste-paléographe, professeur de diplomatique contemporaine. Ainsi, même si Éric de Grolier, qui a enseigné à I'INTD, est considéré comme l'un des fondateurs des sciences de l'information, il est plus sûrement le dernier des encyclopédistes, reconnaissant Diderot pour son « véritable maître à penser » [12].

Voilà résumé l'écartèlement fondateur vécu par la profession. Si on y ajoute le souci d'exister à côté de conservateurs parfois très conservateurs, la nécessaire maîtrise de techniques qui évoluent rapidement, la vocation de pionnier liée à ce métier, on comprend mieux le permanent souci de se faire connaître et reconnaître, dont témoignent à la fois l'action de l'ADBS et les travaux des enseignants-chercheurs de la discipline (voir par exemple [3], [7] et [11]).

Cette reconnaissance est d'autant plus difficile à asseoir qu'il existe aujourd'hui une multiplicité de formations ; cette situation illustre la diversité des pratiques professionnelles et des besoins des employeurs mais grève lourdement la lisibilité du métier. L'ADBS a consacré beaucoup d'efforts à leur recensement et à leur analyse [26]. Là encore, deux traditions s'affrontent : d'une part, la culture générale des apprentis documentalistes qui, du fait de la tradition érudite, doit forcément être élevée; d'autre part, la culture de technicité que l'on s'efforce de valoriser dans un contexte où la technique (celle des ingénieurs, des informaticiens, des médecins) est un gage d'identification et de reconnaissance sociale [20]. Et, pourtant, les documentalistes continuent de faire un travail invisible, s'attachant avec force et conviction à ne montrer de ce qu'ils font que la partie qu'ils estiment valorisante.

Or ranger et travailler aux outils de rangement n'est pas valorisant, c'est au mieux anecdotique. Ce travail invisible, dont on ne peut ni ne veut parler, génère maintes frustrations. À force d'être mésestimé, on se replie sur soi. On en arrive parfois au paradoxe de la documentation fonctionnant pour elle-même, sans plus se préoccuper de ses clients, lesquels bricolent dans leur coin, aggravant encore le phénomène tour d'ivoire d'une documentation parfaite mais inutile [25]. Paradoxe de la société de l'information: la hiérarchie et les utilisateurs méconnaissent le travail des documentalistes mais n'ont de cesse de remplacer les documentalistes par des outils logiciels performants. C'est à la fois reconnaître qu'un certain travail est fait, et qu'on n'y comprend rien. Il est tout aussi tentant pour la hiérarchie et les utilisateurs de n'avoir qu'un seul interlocuteur 
pour toutes les questions documentaires, qu'il s'agisse d'archives, de collections de périodiques, d'albums photos, d'affiches, de documents pédagogiques, des divers rapports des enseignants et des élèves, de l'alimentation d'une base de données bibliographiques ou d'archivage des documents électroniques. La tendance générale est à la fusion, voire à la confusion des activités documentaires, ce qui nuit encore à leur visibilité et complique la qualification du service rendu.

Un dernier aspect rend cette activité invisible : elle fonctionne en effet généralement sans faire de vagues et, lorsqu'un service est supprimé, sa disparition ne semble apparemment pas créer d'irréversible dommage immédiat. La multiplication des banques de données en ligne, désormais conjuguée à la disponibilité des documents en texte intégral au format électronique, a laissé croire un temps que le métier de documentaliste n'était plus indispensable. Entreprises, organisations et documentalistes ont rapidement abandonné cet espoir pour les uns, cette crainte pour les autres [8]. L'heure est aujourd'hui à la médiation documentaire, comme en témoignent colloques et publications professionnels (voir par exemple [10] et [13]). II s'agit de passer d'intermédiaire à médiateur. Mais le médiateur est-il vraiment mieux perçu que le prestataire de service ? Sa position " à côté » des préoccupations du cœur de métier le marginalise, l'isole de ceux qui sont dans l'action. Son travail n'en apparaît ni plus technique, ni plus visible [9].

\section{Un métier de service et ses aléas}

Et pourtant, s'il est un métier qui se situe au cœur des relations entre les personnes, c'est bien celui-ci. Peut-on concevoir un centre de documentation, une bibliothèque, un dépôt d'archives sans « des gens pour s'en servir »- utilisateurs, clients, lecteurs, usagers ou tout simplement chercheurs, médecins, juristes, du nom de leur profession?

Les trois compétences de la relation de service selon Erving Goffman ${ }^{4}$ [17] permettent de réaliser que les difficultés rencontrées pour la documentation sont celles de toute relation de service: - les compétences techniques semblent simples alors qu'elles sont complexes (négocier tel accès au texte intégral de revues électroniques au détriment de tel abonnement papier en pariant sur les évolutions de la politique de conservation électronique des éditeurs, par exemple) ;

- les compétences relationnelles et de civilité sont peu valorisées et pourtant indispensables si l'on veut que le client se sente à l'aise (comment, par exemple, rendre service à la communauté des utilisateurs sans être systématiquement au service de tous les individus ?) ;

- la dimension contractuelle est construite de telle sorte qu'il est toujours difficile de distinguer ce qui est purement professionnel de ce qui est affectif. À titre d'exemple, la prestation documentaire est rarement une dépense liée à un projet. À rester globale, elle est invisible au niveau collectif, tout en laissant un espace au niveau individuel pour les personnes influentes et capables de négocier achats ou abonnements.

Les implications sociales et subjectives apparaissent dans toute relation de service, au-delà de la seule production. Montrer la faible nuance entre service et servitude, c'est compléter les travaux sur l'économie des services (par exemple [14] et [15]) et dépasser le principe d'asymétrie d'information ${ }^{5}$ au profit de celui qui réalise le service, pour être attentif au pouvoir qu'exerce le client: "Dans cet écart qui sépare les actes que le [praticien] consent à faire pour aider le client et les actes qu'il refuse parce que le client peut le faire lui-même, on est précisément au cœur du problème de la servitude [le serviteur fait ce que le maître ne souhaite pas faire] " [23, p. 205]. Par exemple, le documentaliste du Labo que nous étudions ne veut pas être au service de tous les chercheurs car leurs demandes ne lui semblent pas toutes légitimes au regard de la reconnaissance qu'il attend. On retrouve dans toute relation de service ce que vit ce documentaliste qui n'a pas la force d'affirmer sa position: "L'asservissement semble être constitutif de toute relation de service dans les cas où le prestataire ne dispose pas de ressources de prestige et de pouvoir suffisantes pour l'empêcher. Le problème [...]

$4 \quad$ Nous généralisons ici sa définition à une relation qui n'existe pas seulement vis-à-vis d'un public externe mais qui est aussi interne à une organisation, et nous allons au-delà de l'interaction. Insister conjointement sur les relations de service et les compétences est aussi significatif d'une préoccupation contemporaine (voir notamment [19]).

$5 \quad$ Voir la théorie des coûts de transaction en économie [1]. 
est celui de l'usage de soi par soi [...] rendu problématique dans les métiers de service du fait de l'usage qui est fait de soi par autrui. » [25, p. 208]

\section{Du service dû/rendu à la coproduction}

Pour dépasser ces souffrances et passer de la servitude au service, nous mobilisons la notion d' « agencement documentaire ", en référence aux agencements organisationnels de Jacques Girin [16], qui permet de clarifier la relation entre les mandants, ceux qui disent (demandent un service documentaire), et les mandataires, ceux qui font (rendent ce service) par l'intermédiaire d'un mandat. Cette théorie s'inscrit dans un ensemble de courants de recherche : le langage au travail, la cognition distribuée, l'intelligence collective [33], les théories de l'action organisée et la nouvelle économie institutionnelle.

Le mandataire est un composite de ressources: humaines (les personnes qui accomplissent la tâche), symboliques (le langage et en particulier les documents) et matérielles (bureaux, téléphones, ordinateurs, etc.). Pour atteindre une certaine performance précisée par le mandat, ce mandataire doit avoir une certaine compétence. Celle-ci consiste en la capacité à établir des liens entre les ressources et non à mobiliser ces éléments les uns indépendamment des autres. Elle peut être étudiée en détail pour comprendre les défaillances ou au contraire les prévenir et favoriser l'atteinte de la performance.

En effet, le mandat peut être clair ou confus et en conséquence l'activité qui en découle simple ou complexe. Les rendez-vous au cours du mandat sont l'occasion de conversations entre mandant et mandataire, qui permettent d'avancer le plus possible en cohérence. Dans le cas d'un mandat clair, il est possible de spécifier précisément le service attendu et de mettre en place des indicateurs sur les résultats attendus. En revanche, dans le cas d'un mandat confus, les seuls indicateurs possibles portent sur les moyens à mettre en œuvre (limiter les responsabilités, faire des comptes rendus précis ou simplifiés selon la complexité de l'activité, etc.) ; il faut alors attendre la réalisation de l'activité pour juger du résultat. Les problèmes en organisation relèvent le plus souvent de mandats confus et d'activités complexes où la coproduction entre mandant et mandataire est indispensable. Ce qui est le cas dans l'exemple que nous nous proposons d'étudier.

\section{Présentation du cas Labo}

Le Labo nous ${ }^{6}$ a appelées pour l'aider à décider de la survie ou non de sa bibliothèque et nous l'avons aidé à retisser les liens entre la documentation et le travail des chercheurs. Ces derniers sont environ soixante (y compris les professeurs et les thésards), avec une dizaine d'administratifs dont un bibliothécaire et un documentaliste ${ }^{7}$, un chargé de communication et deux informaticiens. Ces cinq dernières personnes forment la population des professionnels de l'information documentaire, certains de façon permanente (bibliothécaire et documentaliste), d'autres de façon ponctuelle (communication et informaticiens).

Le Labo est en phase de repositionnement stratégique et subit en même temps des baisses budgétaires importantes: association entre organisme de recherche et université, ce qui augmente la taille du Labo et modifie le profil des chercheurs (davantage d'enseignants chercheurs), besoin de publier en anglais, nécessité de réorganiser le fonctionnement des programmes de recherche trop éclatés pour les recentrer sur la thématique historique du Labo et améliorer ainsi la visibilité des travaux qui y sont menés. En ce qui concerne les ressources documentaires, les bases de données en sciences humaines et sociales commencent à se multiplier.

La question de la survie de la bibliothèque s'inscrit dans ce double cadre d'évolutions organisationnelles et techniques. La bibliothèque existe depuis l'origine du Labo et les chercheurs y sont attachés, malgré une histoire mouvementée. D'abord gérée par un bibliothécaire jaloux de ses ouvrages (il était presque impossible aux chercheurs d'en sortir un livre), la bibliothèque a ensuite été prise en charge par une ancienne secrétaire

\section{$6 \quad$ Nous sommes nous-mêmes chercheuses et praticiennes.}

7 La distinction n'est pas toujours nécessaire mais, dans le cas présent, l'histoire du Labo et des personnes oblige à séparer les rôles et les fonctions, au moins de façon transitoire (pour plus de détails, voir [32]). 
proche des chercheurs qui assurait une gestion simple et très souple (si souple que, à l'opposé de la première période, beaucoup d'ouvrages ont disparu). Malade durant plusieurs années, la secrétaire n'a été remplacée qu'épisodiquement par des personnes en contrats temporaires et, petit à petit, la bibliothèque a été laissée en friche.

Quatre ans avant notre intervention, un documentaliste diplômé de l'enseignement supérieur a été embauché : il s'est attelé à remettre de l'ordre, à informatiser et à négocier les accès aux banques de données de l'université associée. Très récemment, un bibliothécaire est venu le décharger de la gestion de la bibliothèque. Le documentaliste est donc censé se consacrer à un travail de production de dossiers documentaires en association avec les chercheurs. II reste pourtant relativement isolé et se sent submergé : "Les chercheurs ont l'air plus au courant que moi de l'actualité ", "Ils ne se sont pas approprié la logique documentaire ", "Ils me sollicitent à chaque panne de réseau mais je ne peux rien faire."

Nous sommes présentes dix mois pour étudier les pratiques des chercheurs, analyser l'offre documentaire et proposer des scénarios d'évolution. Nous rencontrons longuement quatorze chercheurs ${ }^{8}$ (sur soixante) ainsi que les professionnels de l'information, nous intervenons sur la bibliothèque notamment pour la mettre sous procédure ${ }^{9}$ et participer au choix de nouveaux logiciels de gestion, et nous animons un comité de pilotage composé des membres de la direction, des responsables de l'information et de la communication et des représentants des chercheurs.

La question initiale de la survie de la bibliothèque passe par l'étude des relations entre le métier du Labo (la recherche) et la documentation. Nous avons choisi de le faire en nous intéressant de très près aux pratiques de travail des uns et des autres. Cette approche est en phase avec ce que nous avons intégré des travaux des sociologues des usages (par exemple [6], [24] et [28]) : un besoin ne s'étudie pas mais une pratique peut être analysée, ainsi que sa cohérence avec des outils existants ou potentiels.

Nos interlocuteurs chercheurs apprécient que nous ne leur posions pas de question sur d'éventuels besoins qu'ils savent ne pas pouvoir exprimer et sont diserts sur leurs pratiques quotidiennes : leur métier, comment ils l'envisagent et le pratiquent, leurs difficultés, leurs relations aux collègues proches ou lointains, leur quête d'information sous toutes ses formes, leur nécessité de communiquer, les affres de la publication et de la mise en forme bibliographique, leurs rangements, etc. On déroule un fil qui part de leur travail pour arriver à la bibliothèque en passant par le documentaliste et Internet. Nous faisons de même pour les membres du secrétariat général et les directeurs (deux personnes se succèderont durant notre intervention).

Un premier niveau d'analyse est alors possible, celui de l'agencement organisationnel qui devient ici l'agencement documentaire.

\section{L'agencement documentaire du Labo}

Le mandat entre la population des chercheurs et la bibliothèque est a priori clair: apporter un service documentaire pour la recherche. Et la tâche de la bibliothèque est a priori simple : gérer le fonds et le flux documentaires (commander, s'abonner, classer, prêter, relancer) et s'informer puis informer sur les outils d'information en ligne.

En fait, la situation n'est pas si nette : le mandat est confus et la tâche complexe. En effet, les chercheurs sont eux aussi, en quelque sorte, des professionnels de l'information : ils trouvent leur documentation à travers leurs propres réseaux (et moins bien dans leur propre rangement), ils se débrouillent parfois seuls avec les outils Internet et ils ont des exigences floues mais fortes en matière de recherche documentaire ponctuelle, d'abonnements et d'achats. Par ailleurs, l'accès aux ressources électroniques (textes intégraux mais aussi bases de données de référence ou localisations documentaires) est soumis aux négociations entre le Labo et ses universités de rattachement ainsi qu'avec sa principale tutelle. L'accès à la bibliothèque du Labo dépend

$8 \quad$ Ce terme générique regroupe la famille suivante: chargé de recherche, maître de conférences, professeur, directeur de recherche, thésard.

$9 \quad$ Nous entendons ici par «mise sous procédure» la description détaillée des tâches élémentaires de chaque activité de la bibliothèque. Ce travail est réalisé par un étudiant de DEA formé à cette pratique, Athissingh Ramrassingh. 
étroitement de la discipline de ses utilisateurs et le rôle de l'informatique et de la communication n'est pas négligeable dans l'offre de service documentaire.

La concertation, voire la négociation, sont ainsi souvent nécessaires. Mais l'organisation correspondante n'est pas pensée puisqu'on se croit en régime de simple service dû/rendu, ce qui engendre un malaise dont nous avons observé quelques manifestations durant notre intervention : la coopération est mal vécue car non prévue (ce n'est jamais le bon moment), les velléités sont courantes ("Je devrais lui demander... ", "Je devrais ranger comme untel ", " II faudrait prévoir des budgets... ") et l'excuse du manque de temps et d'argent pour ne pas coopérer est la règle.

En résumé, la population des chercheurs a du mal à formuler ses demandes en matière documentaire et le " composite documentaire ${ }^{10}$ » est mal pourvu pour y répondre. La réponse est rarement immédiate (un peu de formation serait utile, par exemple, ou bien il faudrait définir un budget, etc.) ou alors elle est considérée comme arbitraire (on ne fournit pas les thésards en photocopies d'articles ou on ne leur procure pas d'ouvrages via le prêt entre bibliothèques, par exemple, ou bien on interrompt l'abonnement à une revue papier indispensable, etc.). On observe ainsi autant un besoin mutuel qu'une crainte réciproque, ce qui peut se manifester par du mépris d'une part et de l'enfermement d'autre part.

\section{Recentrer la documentation au cœur de l'activité du Labo}

Comment améliorer la compétence et la performance de l'agencement documentaire du Labo ?

Au cours du premier comité de pilotage, on recense une série de questions d'apparence simple qui va permettre d'alimenter la suite de notre recherche :

- ouvre-t-on la bibliothèque à un public plus large d'étudiants? Ce qui pose les questions des sources de financement et de la liberté de fonctionnement, ainsi que celle des horaires d'ouverture ; - le chercheur peut-il sous-traiter un travail de recherche documentaire ou doit-il au contraire être formé pour pouvoir l'assurer lui-même?

- comment articuler la démarche du Labo avec la politique du département SHS et celle des partenaires universitaires ? Cela implique d'avoir des partenariats documentaires non seulement locaux, mais également nationaux et internationaux, en matière de fonds documentaire et de compatibilité des logiciels ;

- comment se procurer un document étranger, surtout en anglais, quel que soit le réseau ? Comment juger de la qualité d'un document via Internet?

- Gdocu ${ }^{11}$ semble un bon outil pour gérer ses références par mots clés, usages, auteurs, etc., pour présenter automatiquement ses bibliographies, mais les chercheurs doivent-ils faire le deuil de leur classement artisanal ?

Au quatrième comité de pilotage, les scénarios d'évolution de la documentation en relation avec l'organisation du Labo sont les suivants :

- à un extrême, ne changer que la relation entre les parties prenantes, à savoir développer la formation sur les outils par l'intermédiaire de volontaires, assurer un lien plus systématique entre documentation et communication pour la valorisation de la recherche et moderniser la gestion de la bibliothèque (nouveau logiciel et mise sous procédures). Ce scénario minimal représente déjà un progrès par rapport à l'existant puisqu'il oblige à repenser les liens entre les fonctions et à intégrer un minimum le chercheur (pour la formation notamment) ;

- à l'autre extrême, la bibliothèque disparaît, ce qui oblige à des collaborations intenses : connexions sans failles, imprimantes puissantes, négociation stratégique avec l'université, concertations entre chercheurs et professionnels de la documentation, etc.

10 Ce peut être le lieu bibliothèque et un autre chercheur à un moment donné, le documentaliste et une base de données, un article papier, le réseau informatique et son support technique local ou universitaire, un ouvrage, etc.

11 Ce terme est un néologisme créé pour voiler le nom du logiciel. 
Ces deux scénarios sont impossibles : le premier car il est dommage de se contenter de si peu de progrès alors que le potentiel est plus élevé et les enjeux stratégiques forts ; l'autre car il est utopique en l'état. Restent deux scénarios intermédiaires.

Un scénario qui ressemble au premier mais renforce les actions concertées, à savoir coordonner les budgets de la documentation avec les contrats et les axes de recherche, s'entendre sur les contenus (ouvrages, abonnements papier et électroniques, réseaux, etc.) entre documentaliste, bibliothécaire et chefs de projet. Ce scénario produirait une petite révolution puisqu'il suppose qu'il y a des chefs de projets, alors qu'actuellement la question de la légitimité des responsables de programmes de recherche est posée. Nous signalons qu'une politique documentaire n'a de sens qu'insérée dans une démarche stratégique et que, pour cela, une organisation cohérente du travail est nécessaire. II n'y a là rien de nouveau pour les membres du comité de pilotage car tous ont conscience de l'importance de cette organisation. Ils n'ont en revanche pas les clés de la restructuration. Ils sont intrigués par la notion de politique documentaire.

Un autre scénario intermédiaire ajouterait la dimension enseignement en intégrant à la fois le SUDOC (Système universitaire de documentation), ce qui nécessite une restructuration des données saisies dans le logiciel actuel (et qui ne sont pas déjà au SUDOC, à savoir moins de $5 \%$ des ouvrages et revues), un nouveau logiciel documentaire et l'accueil des étudiants des masters 1 et 2 . Cela pose plus explicitement la question de la spécialisation du fonds.

Tous ces scénarios tiennent compte de la baisse budgétaire et de l'orientation vers des publications en anglais. Les évolutions techniques se font en concertation avec l'informatique et avec les projets des institutions en matière d'édition électronique en sciences humaines (récents par rapport aux autres sciences). Dans le dernier scénario présenté, il s'agit de tirer parti du rapprochement universitaire : intégrer le SUDOC signifie s'engager dans une démarche d'intégration au service commun de documentation de l'université et inciter celle-ci à fournir de la formation et éventuellement du personnel pour assurer le suivi de la base, voire de la bibliothèque. Or le bibliothécaire ne doit rester en poste que quatre ans : c'est l'occasion de pérenniser son poste sans pour autant disposer d'un budget spécifique. C'est aussi une façon de se rapprocher des instances de décision universitaire.

C'est ce dernier scénario qui sera finalement retenu après de longues discussions et remises à plat de l'ensemble des questions. Il aura en effet fallu mettre la bibliothèque sous procédure (pour chiffrer la fréquentation et les saisies ainsi que pour vérifier la cohérence des actions et les cotations), s'appuyer sur l'implication des chercheurs (dont une petite équipe qui prendra notre place de médiatrices après notre départ) et mener une série d'actions à court terme (qui laissent présager du plus long terme comme l'intranet souvent souhaité), etc.

Au cours des comités de pilotage prend également forme l'idée d'un conseil documentaire (rendez-vous réguliers entre mandants et mandataires), instance de décision sur les questions de documentation en lien avec l'activité du Labo. Il s'agit de stimuler l'attention mutuelle des chercheurs et des professionnels de l'information documentaire grâce à des actions concertées rythmées par des rendez-vous précis. On le précise jusque dans le détail de son organisation qui semble en phase avec les pratiques de l'organisation. Le conseil documentaire est entièrement fonction de la politique documentaire que nous aidons à mettre en œuvre et qui tient compte du potentiel d'évolution de la structure telle que nous avons pu l'étudier. La politique documentaire est en lien étroit avec la politique générale (ou stratégie) du Labo.

$C^{\prime}$ est en effet au sens le plus large possible que nous entendons ici la politique documentaire. Ce qui est vrai en matière de politique publique l'est tout autant lorsqu'il s'agit de la politique d'une organisation. Pour reprendre les termes d'Anne-Marie Bertrand [4] : "Quoi de plus politique que l'accès au savoir ? Quoi de plus politique qu'une institution de partage du savoir ? $^{12}$ »

Pour un laboratoire de recherche, parler de stratégie, c'est mettre l'organisation et la production scientifique en cohérence avec les sphères institutionnelles (en l'occurrence les tutelles de rattachement), économiques (les contrats avec le privé, par exemple) et scientifiques (notamment les publications [27]). La stratégie n'est pas forcément explicite, parfois sujette à exercices collectifs réguliers, parfois ignorée et souvent seulement

\footnotetext{
12 Dans le cas qui nous occupe, la politique documentaire dépasse largement le cadre d'une politique d'acquisition.
} 
développée sur une ou deux dimensions de "la rose des vents» de la recherche [5] : favoriser plutôt les publications, l'innovation économique, la formation, la vulgarisation ou l'expertise. Ici il s'agit de multiplier les publications internationales, de réduire l'activité économique et de développer la formation.

La politique documentaire permet de faire le lien entre les aspects opérationnels concernant la documentation et la stratégie du Labo. De façon plus précise, elle prend la forme suivante :

- affirmer le fonds documentaire pour peser dans la négociation avec les autorités de tutelle. Le Labo s'impose par son seul fonds documentaire, a fortiori lorsqu'il se saisit d'une démarche de modernisation et se soucie de cohérence par rapport à son environnement ;

- rendre plus cohérente la politique d'acquisition pour manifester le positionnement scientifique du Labo. Cette politique d'acquisition est source de valorisation des productions et l'un des critères sur lesquels on peut évaluer une unité de recherche ;

- rapprocher les questions documentaires de l'économie du laboratoire : lors de la négociation de contrats de recherche et dans la réponse aux appels d'offres, la bonne performance de cette activité (réactivité, rapidité de traitement, pertinence des résultats de recherche et des produits documentaires) est source de compétitivité.

À partir de la ligne directrice découlant de la politique documentaire, le conseil documentaire (une séance de une heure et demie à deux heures par mois) permet à ses représentants de traiter des questions récurrentes de la documentation en lien avec la communication et l'informatique : accueil des étudiants, formation, achats pour quels projets, mots clés, saisie dans GDocu, procédure d'échanges d'articles et valorisation des travaux du Labo, relance des chercheurs pour mettre à jour les dossiers du Labo, etc. Les représentants des chercheurs préparent les réunions en assurant un relais auprès de leurs collègues pour instruire toutes ces questions (recueillir des besoins, cerner la faisabilité technique, évaluer les coûts, etc.) et préparer les arguments de négociation pour le conseil (notamment pour les achats, les mots clés, les saisies ou les valorisations de travaux).

\section{Conclusion : les rôles de l'agencement documentaire}

En mettant en place une politique documentaire grâce au conseil documentaire, le Labo, en douceur, a réconcilié stratégie et documentation: chacun prend sa part de travail ancillaire (par exemple, pour les chercheurs, saisir leur bibliographie et leurs publications ; ou, pour les documentalistes, vérifier la cohérence budgétaire des achats et la disponibilité des ouvrages), ce qui favorise la collaboration des chercheurs et des professionnels de la documentation et facilite la prise de décision (achat, ouverture de la bibliothèque, banques de données, etc.). Ce partage des tâches est possible grâce à la présence de deux personnages primordiaux : le directeur, qui s'implique et tranche lorsque c'est nécessaire, et les « batteurs de couloir » [31], qui relancent les chercheurs sur toutes les questions documentaires (quels achats faut-il programmer pour tel projet ou tel axe de travail ? qui doit contribuer à la saisie des documents de tel rapport, etc.). L'agencement documentaire sert alors non seulement à mieux travailler mais à mieux le faire ensemble; il permet aussi de montrer la qualité du travail effectué et pas uniquement à rassurer le collectif par sa présence.

Le schéma ci-dessous illustre une analyse de l'agencement documentaire par les rôles [30].

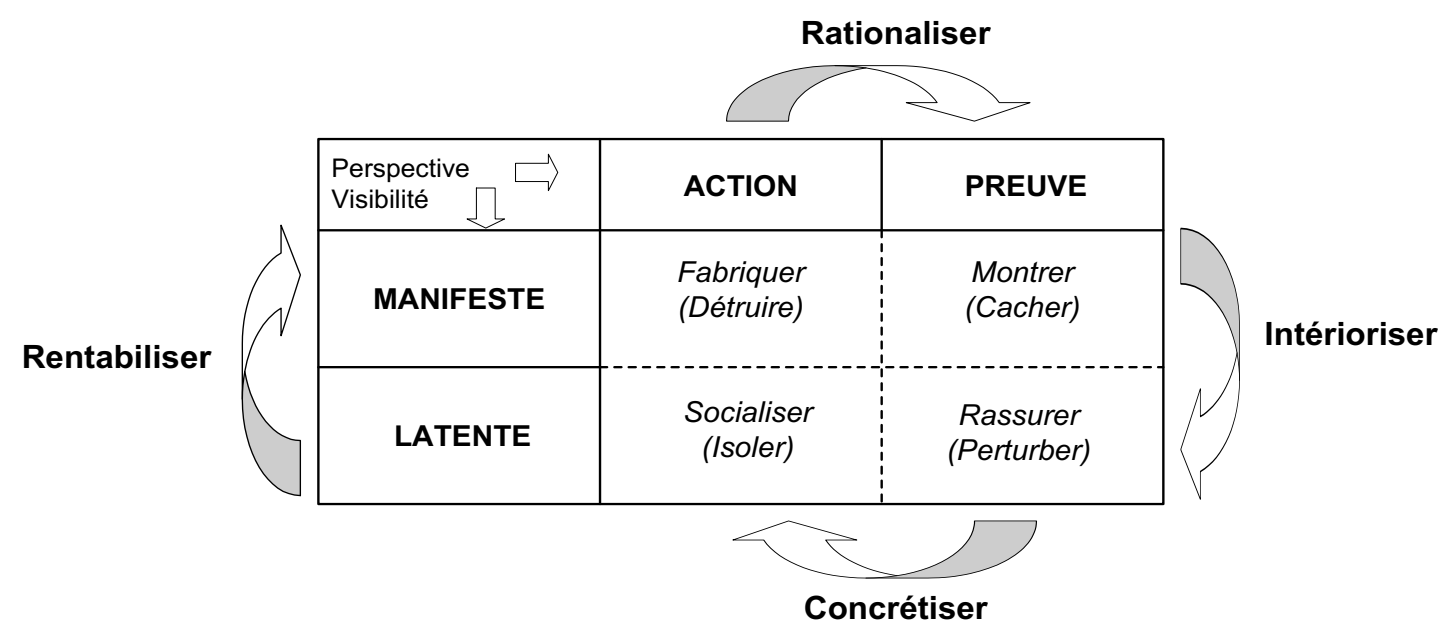


Grille dynamique des fonctions de l'agencement documentaire ${ }^{13}$

Le Labo est passé d'une situation "fébrile " (où l'information sert à fabriquer et à rassurer mais n'est pas intégrée dans la stratégie à long terme) à une situation cohérente où l'information qui rassure (avoir une bibliothèque ne signifie pas forcément qu'on s'en sert, mais sa présence rassure) peut aussi être facteur de socialisation, de performance (l'information mieux ciblée grâce à la coproduction est plus pertinente) et être une vitrine de la stratégie, ce qui permet notamment la négociation de ressources avec les tutelles. Notre travail d'intervenant a été d'aider le Labo à travailler sur les quatre registres : rationaliser (mise sous procédure de la bibliothèque, par exemple), concrétiser (partager les tâches ancillaires), rentabiliser (la politique documentaire précise l'accueil d'étudiants, les prêts entre bibliothèques, le budget documentaire sur chaque projet, etc.), intérioriser (coopérer régulièrement grâce au conseil documentaire).

D'une façon générale, pour la documentation, s'immiscer au cœur de la stratégie d'une organisation, c'est tirer parti de l'invisible matérialité du travail quotidien d'information ${ }^{14}$ en développant une reconnaissance mutuelle. La discrétion reste de mise. II ne s'agit pas d'édicter de grands principes ou d'augmenter la technicité du travail mais de porter un regard neuf sur la distribution des tâches, des personnes et des savoirs. Les efforts correspondants (s'atteler plus souvent qu'auparavant et explicitement à des tâches matérielles et considérées comme subalternes) ont des avantages bien tangibles : outre le fait d'améliorer l'efficacité collective, partager le travail de manutention d'information rapproche les acteurs et peut être mis en valeur (un dossier documentaire résultant du travail conjoint d'un praticien et d'un documentaliste, par exemple). En insistant sur ce qui fait la matérialité du service et sur la nécessaire organisation correspondante, nous avons voulu montrer concrètement ce qui est commun à tous et peut ainsi fonder des enjeux collectifs sans chercher à fixer des buts ou à construire des savoirs communs sophistiqués.

\section{RÉFÉRENCES}

[1] ABÉCASSIS C. « Les coûts de transaction : état de la théorie ». Réseaux, vol. 15, juillet-août 1997, n 84, p. 919

[2] BARRET-KRIEGEL B. Les historiens et la monarchie. Paris : Presses universitaires de France, 1988. 4 vol.

[3] BATTISTI M., FERCHAUD B., et al. " Un métier, des métiers : convergences et spécificités des métiers des archives, des bibliothèques et de la documentation " (Journées interprofessionnelles AAF, ABF, ADBS, ADBU, Paris, 28-29 janvier 2005). Documentaliste - Sciences de l'information, février 2005, vol. 42, n 1, p. $48-57$

[4] BERTRAND A.-M. «Bibliothèque, politique et recherche ». Bulletin des bibliothèques de France, 2005, t. 50, $\mathrm{n}^{\circ} 2$, p. $35-40$

[5] CALLON M. "Laboratoires, réseaux et collectifs de recherche ». In: P. Mustar et H. Penan (dir.). Encyclopédie de l'innovation. Paris : Économica, 2003. P. 693-722

[6] CHAMBAT P. "Usages des technologies de l'information et de la communication (TIC) : évolution des problématiques ». Technologies de l'information et société, 1994, vol. 6, n 3, p. 249-269

[7] CHARTRON G. (dir.). Les chercheurs et la documentation numérique : nouveaux services et usages. Paris : Éditions du Cercle de la librairie, 2002. $268 \mathrm{p}$.

[8] CHRISTOPHE L. " L'utilisateur et l'accès aux réseaux: quelles conséquences sur la médiation documentaire ? ». Documentaliste - Sciences de l'information, mars-avril 1997, vol. 34, n² 2, p. 109-112

13 Les fonctions inverses du schéma ne sont pas mobilisées explicitement ici : elles viennent généralement renforcer l'absence de fonction positive, mais elles sont aussi importantes pour relativiser une approche positiviste. Ces fonctions négatives sont toujours plus ou moins présentes.

14 Ou activité d'information, ce que nomme très justement Brigitte Guyot [18]. Cette recherche sur l'importance des tâches matérielles et invisibles est complémentaire des travaux sur l'invisibilité de la matière des objets de travail comme les TIC [21, 22]. 
[9] COLLARD D. "La médiation: une compétence ingérable? ». Les Annales des mines, série Gérer et comprendre, juin 2003, $n^{\circ} 72$, p. 15-25

[10] COUZINET V. Médiations hybrides : le documentaliste et le chercheur en sciences de l'information. Paris : ADBS Éditions, 2001. 340 p. (Sciences de l'information. Série Recherches et documents)

[11] COUZINET V. et RÉGIMBAUD G. (dir.). Recherches récentes en sciences de l'information : convergences et dynamiques. Paris : ADBS Éditions, 2002. 418 p. (Sciences de l'information. Série Recherches et documents)

[12] FAYET-SCRIBES. "La passion de l'organisation des connaissances. Entretien avec Éric de Grolier ». Documentaliste - Sciences de l'information, novembre-décembre 1996, vol. 33, nº 6, p. 286-293

[13] FERCHAUD B. "Médiations et technologies de l'information : regards croisés " (Colloque ADBS, Paris, 2324 octobre 2003). Documentaliste - Sciences de l'information, décembre 2003, vol. 40, nº 6, p. 392-395

[14] GADREY J. Services : la productivité en question. Paris : Desclée de Brouwer, 1996. 358 p.

[15] GALLOUJ F. «Innovation dans une économie de service ». In : P. Mustar et H. Penan (dir.). Encyclopédie de l'innovation. Paris : Économica, 2003. P. 109-130

[16] GIRIN J. " Les agencements organisationnels ». In: F. Charue-Duboc (dir.). Des savoirs en action: contributions de la recherche en gestion. Paris : L'Harmattan, 1995. P. 233-279

[17] GOFFMAN E. Asiles : études sur la condition sociale des malades mentaux. Paris : Éditions de Minuit, 1968. $441 \mathrm{p}$. (Le sens commun)

[18] GUYOT B. « Une activité de travail méconnue : l'activité d'information ». Communication au troisième colloque ICUST, Usages et utilisateurs d'Internet, 12-14 juin 2001. Accessible sur http://archivesic.ccsd.cnrs.fr

[19] IRIBARNE A. (d'). "Santé au travail et entreprise: quand les transformations des normes de travail, d'emploi et de compétences deviennent de nouvelles sources de maladies professionnelles ». In : H. Poltier et al. Travail et fragilisation mentale. Lausanne : Payot, 2004. 26 p.

[20] IRIBARNE P. (d'). La logique de I'honneur: gestion des entreprises et traditions nationales. Paris : Seuil, 1989. $280 \mathrm{p}$.

[21] JEANNERETY. Y a-t-il (vraiment) des technologies de l'information? Lille: Presses universitaires du Septentrion, 2000. $134 \mathrm{p}$.

[22] JEANNERETY. "Les politiques de l'invisible: du mythe de l'intégration à la fabrique de l'évidence ». Document numérique, 2001, vol. 5, n¹-2, p. 155-180

[23] JEANTET A. « "À votre service !" La relation de service comme rapport social ». Sociologie du travail, 2003, $n^{\circ} 45$, p. $191-209$

[24] JOUËT J. « Retour critique sur les usages ». Réseaux, 2000, vol. 18, n 100, p. 490-521

PEREC G. Penser/Classer. Paris : Hachette, 1985. 184 p. (Textes du XXe siècle)

[25] POMART P.-D. « Georges Perec et la documentation : question à notre métier ». Documentaliste - Sciences de l'information, novembre-décembre 1992, vol. 29, nº 6, p. 243-249

[26] PRÉVOT-HUBERT M. "Les professionnels de l'information en France: de la formation initiale à la formation continue jusqu'à la certification professionnelle ". Documentaliste - Sciences de l'information, juin 2004, vol. 41, n³, p. 182-186

[27] RAFFALLI C. Le déséquilibre perpétuel ou comment gérer un laboratoire de recherche. Thèse de doctorat en sciences de gestion, École polytechnique, 27 mai 1998. 261 p.

[28] SAINT-LAURENT A.-F. (de). Informatique en réseau et dynamique des usages. Thèse de doctorat en sciences de l'information et de la communication, Université de Rennes 2, 15 décembre 1998. 294 p.

[29] VACHER B. « Les enjeux de la manutention de l'information ». Système d'information et management, juin 1998, p. 29-38

[30] VACHER B. "Reconnaître le rôle stratégique des activités quotidiennes d'information et de communication : une grille de lecture ». Soumission au débat Org\&Co, Groupe d'études et de recherches sur les communications organisationnelles, bulletin de février 2006, p. 2-8. Accessible sur http://portail.sfsic.org

[31] VACHER B., BELIN O. « La documentation : service ou médiation ? Le cas d'une bibliothèque de recherche en mutation ». Colloque Médiation et ingénierie des connaissances (CoMetiC), Marseille, 14-16 décembre 2003 
[32] VACHER B., LE BIS I., HASSANALYP. " Rôle de l'agencement documentaire : relation entre pratiques prosaïques et stratégie. Le cas exemplaire du "Labo SHS" ». In : A.-F. de Saint-Laurent (dir.). Les collectifs de travail à l'épreuve des technologies de l'information et de la communication. À paraître

[33] WEICK K. E., ROBERTS K. H. "Collective mind in organisations: heedful interrelating on flight decks ». Administrative Science Quarterly, September 1993, vol. 38, n³, p. 357-381

\section{Biographie des auteures}

Isabelle Le Bis est archiviste paléographe et conservateur des bibliothèques. Après avoir été chef de la section Médecine Xavier Bichat de l'Université Paris-7, chargée du développement de l'information médicale pour EDF et Gaz de France et chargée de l'analyse des fonctions d'information au sein d'EDF, elle est actuellement responsable de l'unité Documentation et archives multimédia de l'École nationale de la santé publique (ENSP) à Rennes. isabelle.lebis@wanadoo.fr

Béatrice Vacher est enseignant chercheur en gestion et en sciences de l'information et de la communication. Auparavant en poste en écoles des Mines (Albi et Alès), elle est actuellement chercheur associé au Laboratoire de valorisation de l'information et de la communication du Groupe d'étude et de recherche en sciences de I'information et de la connaissance (LVIC-GERSIC) de I'Université Aix-Marseille-3 et habite en Espagne. Ses travaux consistent à améliorer la compréhension du fonctionnement des organisations et leur potentiel d'évolution à travers l'étude des activités d'information et de communication en lien avec la stratégie. Elle est notamment l'auteure de l'ouvrage La gestion de l'information en entreprises : enquête sur l'oubli, l'étourderie, la ruse et le bricolage organisés, publié en 1997 aux éditions de l'ADBS. beatricevacher@telefonica.net

Les auteures remercient Parina Hassanaly, du LVIC-GERSIC, pour leur avoir permis d'accéder à ce terrain d'expérimentation et pour avoir participé à leur réflexion avec tout son savoir sur la question. 